\title{
Bilateral cerebellar dysfunctions in a unilateral meso-diencephalic lesion
}

\author{
D V O N C R A M O N \\ From the Max-Planck-Institute for Psychiatry, Munich, Germany
}

S U M MARY The clinical syndrome of a 65-year-old patient with a slit-shaped right-sided mesodiencephalic lesion was analysed. A cerebellar syndrome with limb-kinetic ataxia, intention tremor and hypotonicity in all extremities as well as ataxic dysarthria was found. The disruption of the two cerebello-(rubro) - thalamic pathways probably explained the signs of bilateral cerebellar dysfunction. The uncrossed ascending limb of the right, and the crossed one of the left brachium conjunctivum may have been damaged by the unilateral lesion extending between caudal midbrain and dorsal thalamus.

Most of the fibres which constitute the superior cerebellar peduncle leave the cerebellum and originate in cells of the dentate nucleus but also arise from neurons of the globose and emboliforme nuclei. The crossed ascending fibres of the brachia conjunctiva constitute the major outflow from the cerebellum, they form the cerebello(rubro)-thalamic and dentato-thalamic tracts. ${ }^{1}$ Lesions of the superior cerebellar peduncle lead to the same abnormalities as are seen following destruction of hemispheric portions of the posterior cerebellar lobe. Monkeys with section of both cerebellar peduncles show a considerable ataxia, hypotonicity and marked action tremor. ${ }^{2}$ We report a patient with bilateral cerebellar dysfunction, caused by a unilateral meso-diencephalic lesion possibly affecting both superior cerebellar peduncles.

\section{Case report}

On 31 January 1979 a 65 -year-old white male suddenly became unconscious. After several minutes he regained consciousness. He seemed confused and was not able to utter a sound, although he understood simple commands such as "open your eyes," or "do you want something to drink?" He was admitted to a

Address for reprint requests: PD Dr D von Cramon, Max-PlanckInstitut für Psychiatrie, Neuropsychologische Abteilung, Kraepelinstrasse 10, D-8000 München 40.

Accepted 4 February 1981 general hospital where neurological examination showed bilateral miosis, convergent strabism, vertical gaze paresis on upward gaze with gaze-paretic nystagmus, flaccid sensori-motor hemiparesis with increased stretch reflexes and Babinski sign on the left side, and dysmetric movements of the right upper extremity. The CT scan showed an acute haemorrhage in the right mesodiencephalic area. On 19 February 1979 the patient was admitted to our department. We found an isolated paresis of the left superior oblique muscle (the bilateral miosis, convergent strabism and paresis of upward gaze had disappeared). There was a sharp contrast between the monoplegia of the left arm, the slight motor deficit in the left leg, and the normal face. Stretch reflexes were increased, and Babinski's sign was present, on the left side; stretch reflexes on the right side were reduced. Appreciation of touch was reduced on the left upper extremity sparing the face; appreciation of pain was diminished in the left arm and face; positional sense was lost in the left hand only; vibratory sense and appreciation of temperature was impaired on the entire left half of the body. Tone was markedly reduced in all extremities; elbow- and knee-joints could easily be hyperextruded. Because of "weakness" the patient could not keep himself upright without support. He was not able to walk because of a severe gait ataxia. Coarse dysmetric movements of the non-paretic extremities rendered the patient completely helpless.

By March 1979 the weakness of the left arm had improved, but dysmetria movement now was evident, and there was bilateral coarse intention tremor and pathological rebound. Verbal communication was hampered by dysarthric and dysphonic speech; the 
voice was breathy with voice breaks and diplophonic intervals. Articulation was characterised by a marked slowness of articulatory movements, reduced tightness of constrictions and the inability to achieve high tongue elevation in the production of vowels and consonants. Features of disordered prosody were slow speech rate, reduced modulation of pitch and intensity as well as an equalisation of syllable length. Furthermore, excess and equal stress on ordinarily unstressed syllables occurred. There was, however, no indication of an aphasic disorder. Apart from a grotesque ataxic macrographia, handwriting was not disturbed. Subsequently the patient complained of burning pain in the left arm. Clinical examination revealed hyperpathia of the left arm sparing the hand. This symptom disappeared within a few days.

Selective angiography of the left and right vertebral arteries revealed no abnormality. Repeated EEGs did not show any abnormality. The CSF was normal. CT-scan in March 1979, showed a small hypodense lesion in the right meso-diencephalic region (figure).

In the figure six details of the meso-diencephalic area are shown in a sequence from left-up to rightdown. The upper left detail corresponds to a "section" of the brain stem through the inferior colliculi (at the transition to the rostral pons). At the level of the
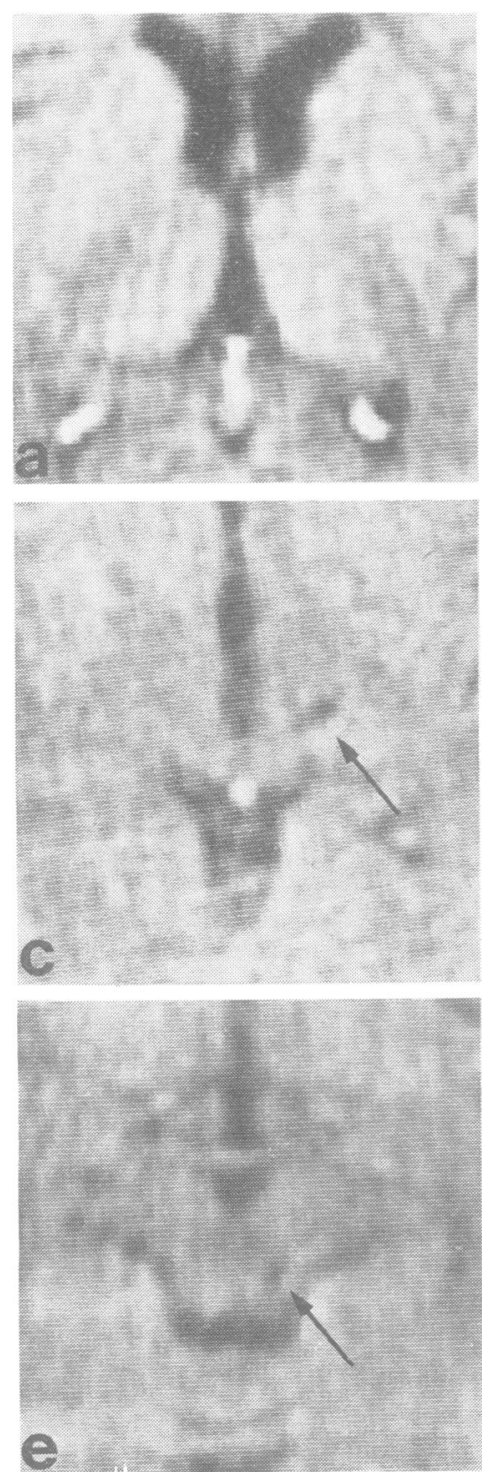
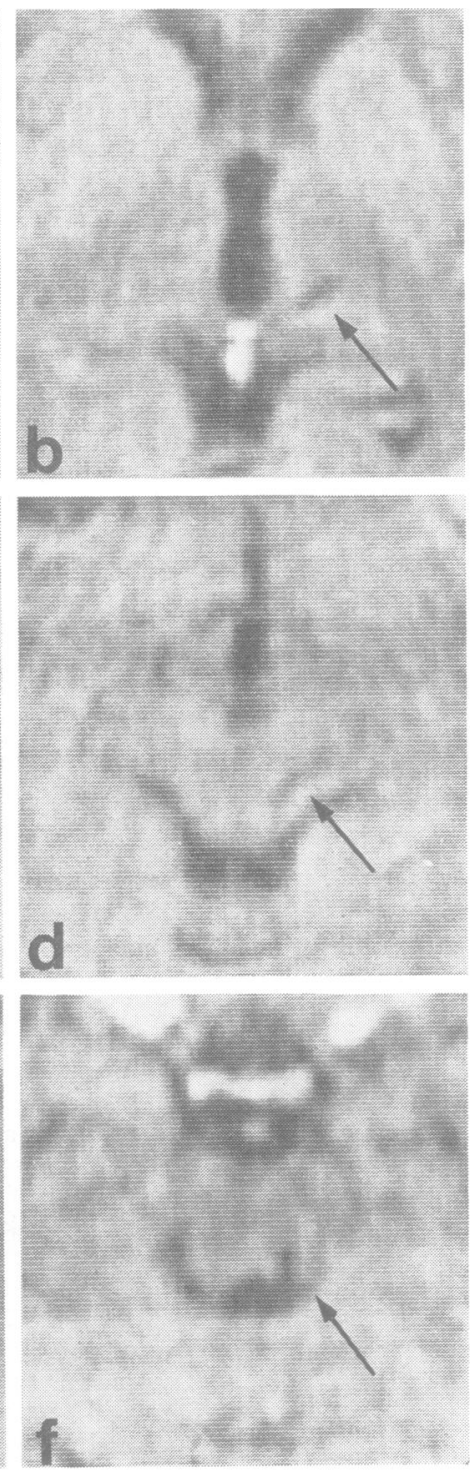

Figure CT scan. Six details of the meso-diencephalic area in a sequence from left up to right down. The arrows indicate the site of the lesion. 
inferior colliculus the hypodense lesion seemed to reach its most caudal extension. At levels through the rostral midbrain the lesion affected the lateral tegmentum sparing the superior colliculus. At the transition from midbrain to diencephalon it extended into the lateral part of the pedunculus. Passing through the ventral thalamus (subthalamic area) it ended in the posterior and basal region of the dorsal thalamus. The internal capsule was not affected. The hypodense lesion may have been a residual necrosis after the resorption of a spontaneous haemorrhage.

\section{Discussion}

The patient's most salient clinical features resembled a cerebellar syndrome. As in lesions of the posterior cerebellar lobe limb-kinetic ataxia, action tremor and hypotonicity in all extremities were observed. ${ }^{2}$ Paresis of the left arm temporarily lessened the degree of dysmetria in this extremity.

In a unilateral mesencephalic lesion, ataxia homolateral to the lesion would be expected, if the superior cerebellar peduncle is injured before its decussation in the caudal midbrain, explaining the dysmetria and intention tremor on the right side in this patient; a lesion of the right superior cerebellar peduncle before its decussation is supported by the CT-scan. The lesion reached down to the ponto-mesencephalic transition caudal to the decussation area, explaining the paresis of the left superior oblique muscle due to involvement of the fibres of the right trochlear nerve before its decussation. ${ }^{3}$

After the paresis of the left arm had receded, dysmetria and intention tremor became evident in the left upper extremity, suggesting damage to the left superior cerebellar peduncle; the CT scan suggested that the ascending crossed limb of the left superior cerebellar peduncle was likely to be affected in the lateral midbrain tegmentum. There was no indication that the decussation area itself had been injured; the crossed descending limb of the left brachium conjunctivum should have been preserved.

The characteristics of the speech disorder in this patient were consistent with the so-called ataxic dysarthria frequently seen following cerebellar lesions..$^{-6}$ Such an ataxic dysarthria may apparently also occur as a result of a lesion of the superior cerebellar peduncles. Lechtenberg and Gilman $^{7}$ claimed that cerebellar speech functions were most commonly compromised by damage to the left cerebellar hemisphere. Thus, a lesion of the crossed ascending limb of the left superior cerebellar peduncle should account for the occurrence of ataxic dysarthria. According to these authors the dominance of the left cerebellar hemisphere in the regulation of speech may derive from access of this hemisphere to the nondominant (right) cerebral hemisphere. The access of cerebellar efferent fibres to the right cerebral hemisphere has certainly been disrupted in our patient.

\section{References}

1 Williams PL, Warwick R. Functional Neuroanatomy of Man. Edinburgh: Churchill Livingstone, 1975.

2 Chambers WW, Sprague JM. Functional localisation in the cerebellum, part I. J Comp Neurol 1955; 103:105-29.

3 Crosby EC, Humphrey T, Lauer EW. Correlative anatomy of the nervous system. New York: The Macmillan Co, 1962.

4 Brown JR, Darley FL, Aronson AE. Ataxic Dysarthria. Int J Neurol 1970; 7:302-18.

5 Kent RD, Netsell R. A case study of an ataxic dysarthric: cineradiographic and spectrographic observations. J Speech Hear Disord 1975; 40: 115-33.

6 Kent RD, Netsell R, Abbs JH. Acoustic characteristics of dysarthria associated with cerebellar disease. J Speech Hear Res 1979; 27:627-48.

7 Lechtenberg R, Gilman S. Speech disorders in cerebellar disease. Ann Neurol 1978; 3:285-90. 